\title{
SEMIORTHOGONAL DECOMPOSITIONS FOR TWISTED GRASSMANNIANS
}

\author{
SANGHOON BAEK \\ (Communicated by Harm Derksen) \\ Abstract. In this article, we present semiorthogonal decompositions for \\ twisted forms of grassmannians.
}

\section{INTRODUCTION}

A basic way to study a derived category of coherent sheaves is to decompose it into simpler subcategories and this can be implemented by using the notion of semiorthogonal decomposition [4].

In [6] Orlov gave the semiorthogonal decompositions for projective, grassmann, and flag bundles, which generalize the full exceptional collections on the corresponding varieties by Bellinson [1] and Kapranov [5].

In the case of projective bundles, Bernardara [2] extended the semiorthogonal decomposition to the twisted forms. In this paper, we present, in a similar way, semiorthogonal decompositions for twisted forms of grassmannians. The proof of the main theorem uses the study of $K$-theory of twisted grassmannians by LevineSrinivas-Weyman [7] and Panin [8].

The paper is organized as follows. In Section 3, we recall the notion of semi orthogonal decomposition. In Section 4, we provide the main result (Theorem 4.2) and its application (Corollary 4.3).

\section{Notation AND CONVEnTiOns}

In this paper, a partition means a nonincreasing sequence of numbers. Given a partition $\alpha=\left(\alpha_{i}\right)$, the conjugate of $\alpha$, written by $\alpha^{*}$, is the partition $\left(\alpha_{j}^{*}\right)$, where $\alpha_{j}^{*}=\left|\left\{i \mid \alpha_{i} \geq j\right\}\right|$. For a scheme $X$ over $Y$, we denote by $\Delta(X / Y)$ the diagonal of $X \times_{Y} X$. We abbreviate $\mathscr{F} \otimes \mathscr{G}=\pi_{1}^{*} \mathscr{F} \otimes \pi_{2}^{*} \mathscr{G}$ for sheaves $\mathscr{F}, \mathscr{G}$ over $X$, where $\pi_{i}$ is the projection of $X \times_{Y} X$. For a scheme $X$, we denoted by $\mathrm{D}(X)$ the bounded derived category of coherent sheaves on $X$.

\section{Preliminaries}

In this section we recall some definitions from [3]. Let $F$ be a field. We fix a triangulated $F$-linear category $\mathcal{T}$. Given a full subcategory $\mathcal{S}$ of $\mathcal{T}$, the (right)

Received by the editors March 11, 2013.

2010 Mathematics Subject Classification. Primary 14C35, 14F05; Secondary 18E30, 14M15. 
orthogonal complement, denoted by $\mathcal{S}^{\perp}$, is a full subcategory whose objects are objects $T$ in $\mathcal{T}$ satisfying

$$
\operatorname{Hom}_{\mathcal{T}}(S, T)=0
$$

for all $S \in \mathcal{S}$.

A full triangulated subcategory $\mathcal{S}$ of $\mathcal{T}$ is (right) admissible if for any $T \in \mathcal{T}$ there is a distinguished triangle

$$
S \rightarrow T \rightarrow S^{\perp} \rightarrow S[1]
$$

with $S \in \mathcal{S}$ and $S^{\perp} \in \mathcal{S}^{\perp}$, i.e., $\mathcal{T}=\left\langle\mathcal{S}, \mathcal{S}^{\perp}\right\rangle$.

Now, we consider a sequence $\left(\mathcal{S}_{1}, \ldots, \mathcal{S}_{n}\right)$ of admissible subcategories of $\mathcal{T}$. This sequence is called semiorthogonal if

$$
\mathcal{S}_{i} \subset \mathcal{S}_{j}^{\perp}
$$

for $1 \leq i<j \leq n$. A semiorthogonal sequence $\left(\mathcal{S}_{1}, \ldots, \mathcal{S}_{n}\right)$ of $\mathcal{T}$ is called semiorthogonal decomposition if $\mathcal{T}=\left\langle\mathcal{S}_{1}, \ldots, \mathcal{S}_{n}\right\rangle$.

For example, the sequence $\left(\mathcal{S}, \mathcal{S}^{\perp}\right)$ for an admissible full triangulated subcategory $\mathcal{S}$ gives a semiorthogonal decomposition of $\mathcal{T}$. By definition, any generating sequence $\left(\mathcal{S}_{1}, \ldots, \mathcal{S}_{n}\right)$ of full subcategories of $\mathcal{T}$ satisfying (11) is a semiorthogonal decomposition of $\mathcal{T}$.

\section{TWisted gRassmannians}

Let $X$ be a Noetherian scheme of characteristic 0 and let $\mathscr{A}$ be a sheaf of Azumaya algebras of rank $n^{2}$ over $X$. For an integer $1 \leq k<n$, a twisted grassmannian $p: \operatorname{Gr}(k, \mathscr{A}) \rightarrow X$ is defined by the representable functor from the category Schemes $/ X$ of schemes over $X$ to the category of Sets of sets given by $(Y \stackrel{\phi}{\rightarrow} X) \mapsto$ the set of sheaves of left ideals $\mathscr{I}$ of $\phi^{*} \mathscr{A}$ such that $\phi^{*} \mathscr{A} / \mathscr{I}$ is a locally free $\mathscr{O}_{Y^{-}}$ module of rank $n(n-k)$.

There is an étale covering $i: U \rightarrow X$ and a locally free sheaf $\mathscr{E}$ of rank $n$ over $U$ with the following pullback diagram:

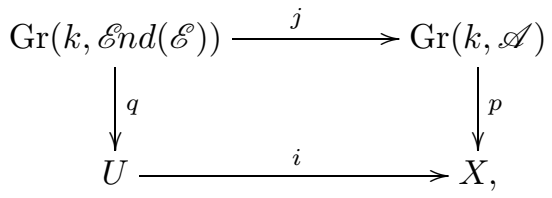

where $\operatorname{Gr}(k, \mathscr{E} n d(\mathscr{E}))$ is naturally isomorphic to $\operatorname{Gr}(k, \mathscr{E})$.

Consider the tautological exact sequence of sheaves on $\operatorname{Gr}(k, \mathscr{E})$

$$
0 \rightarrow \mathscr{R} \rightarrow q^{*} \mathscr{E} \rightarrow \mathscr{T} \rightarrow 0,
$$

where $\operatorname{rank}(\mathscr{R})=k$. For a partition $\alpha=\left(\alpha_{1}, \ldots, \alpha_{k}\right)$ with $0 \leq \alpha_{i} \leq n-k$, we denoted by $S^{\alpha}$ the Schur functor for $\alpha$. We define $S(\alpha)$ to be the full subcategory of $\mathrm{D}(\operatorname{Gr}(k, \mathscr{A}))$ generated by $\mathscr{M}$ in $\mathrm{D}(\operatorname{Gr}(k, \mathscr{A}))$ satisfying

$$
\left.\mathscr{M}\right|_{\operatorname{Gr}(k, \mathscr{E})} \simeq q^{*} \mathscr{N} \otimes S^{\alpha} \mathscr{R},
$$

for some $\mathscr{N} \in \mathrm{D}(U)$.

Lemma 4.1. Let $\alpha, \alpha^{\prime}$ be two distinct partitions with $0 \leq \alpha_{i}, \alpha_{i}^{\prime} \leq n-k$ and let $\mathscr{M} \in S(\alpha)$ and $\mathscr{M}^{\prime} \in S\left(\alpha^{\prime}\right)$. Then $\operatorname{RH}$ om $\left(\mathscr{M}, \mathscr{M}^{\prime}\right)=0$. 
Proof. Let $\left.\mathscr{M}\right|_{\operatorname{Gr}(k, \mathscr{E})} \simeq q^{*} \mathscr{N} \otimes S^{\alpha} \mathscr{R}$ and $\left.\mathscr{M}^{\prime}\right|_{\operatorname{Gr}(k, \mathscr{E})} \simeq q^{*} \mathscr{N}^{\prime} \otimes S^{\alpha^{\prime} \mathscr{R}}$.

By the Littlewood-Richardson rule, the partition $\beta$ of an irreducible summand $S^{\beta} \mathscr{R}$ of $R \mathscr{H}$ om $\left(S^{\alpha} \mathscr{R}, S^{\alpha^{\prime} \mathscr{R}}\right)$ is of the form $\left(\beta_{1}, \ldots, \beta_{k}\right)$ with $-(n-k) \leq \beta_{i} \leq n-k$. Hence, by the Borel-Bott-Weil theorem as in [5, Lemma 3.2] we have

$$
R q_{*}\left(\mathscr{H} \circ m\left(S^{\alpha} \mathscr{R}, S^{\alpha^{\prime}} \mathscr{R}\right)\right)=0 .
$$

It is enough to show the result locally. By the adjoint property of $R q_{*}$ and $q^{*}$, projection formula, and (2), we have

$$
\begin{aligned}
R \mathscr{H} o m\left(\left.\mathscr{M}\right|_{\operatorname{Gr}(k, \mathscr{E})},\left.\mathscr{M}^{\prime}\right|_{\operatorname{Gr}(k, \mathscr{E})}\right) & =R \mathscr{H} o m\left(q^{*} \mathscr{N}, q^{*} \mathscr{N}^{\prime} \otimes \mathscr{H} o m\left(S^{\alpha} \mathscr{R}, S^{\alpha^{\prime} \mathscr{R}}\right)\right) \\
& =R \mathscr{H o m}\left(\mathscr{N}, R q_{*}\left(q^{*} \mathscr{N}^{\prime} \otimes \mathscr{H} o m\left(S^{\alpha} \mathscr{R}, S^{\alpha^{\prime} \mathscr{R}}\right)\right)\right) \\
& =R \mathscr{H} o m\left(\mathscr{N}, \mathscr{N}^{\prime} \otimes R q_{*}\left(\mathscr{H} o m\left(S^{\alpha} \mathscr{R}, S^{\alpha^{\prime} \mathscr{R}}\right)\right)\right) \\
& =0 .
\end{aligned}
$$

Theorem 4.2. Let $\left(S(\alpha) \mid \alpha=\left(\alpha_{1}, \ldots, \alpha_{k}\right), 0 \leq \alpha_{i} \leq n-k\right)$ be a sequence of the full subcategories of $\mathrm{D}(\operatorname{Gr}(k, \mathscr{A}))$ by the lexicographical order on $\alpha$. Then this sequence gives a semiorthogonal decomposition of $\mathrm{D}(\operatorname{Gr}(k, \mathscr{A}))$.

Proof. By Lemma 4.1, it suffices to show that $(S(\alpha))$ generates $\mathrm{D}(\operatorname{Gr}(k, \mathscr{A}))$. Following [7] or [8], there exist sheaves $\mathscr{F}_{\alpha}$ of right $\mathscr{A}^{\otimes|\alpha|}$-modules and sheaves $\mathscr{G}_{\alpha^{*}}$ of left $\mathscr{A}^{\otimes|\alpha|}$-modules such that

$$
j^{*} \mathscr{F}_{\alpha} \simeq S^{\alpha} \mathscr{R} \otimes q^{*}\left(\left(\mathscr{E}^{*}\right)^{\otimes|\alpha|}\right), j^{*} \mathscr{G}_{\alpha^{*}} \simeq q^{*}(\mathscr{E} \otimes|\alpha|) \otimes S^{\alpha^{*}} \mathscr{T}^{*} .
$$

Moreover, the sequence $\mathscr{R} \otimes \mathscr{T}^{*} \rightarrow \mathcal{O}_{\operatorname{Gr}(k, \mathscr{E}) \times \operatorname{Gr}(k, \mathscr{E})} \rightarrow \mathcal{O}_{\Delta(\operatorname{Gr}(k, \mathscr{E}) / U)} \rightarrow 0$ descends to the sequence $\mathscr{F}_{(1)} \otimes \mathscr{G}_{(1)} \rightarrow \mathcal{O}_{\operatorname{Gr}(k, \mathscr{A}) \times \operatorname{Gr}(k, \mathscr{A})} \rightarrow \mathcal{O}_{\Delta(\operatorname{Gr}(k, \mathscr{A}) / X)} \rightarrow 0$. Hence, we have the Koszul resolution:

$$
\begin{gathered}
0 \rightarrow \Lambda^{k(n-k)}\left(\mathscr{F}_{(1)} \otimes \mathscr{G}_{(1)}\right) \rightarrow \Lambda^{k(n-k)-1}\left(\mathscr{F}_{(1)} \otimes \mathscr{G}_{(1)}\right) \rightarrow \cdots \\
\ldots \rightarrow \mathscr{F}_{(1)} \otimes \mathscr{G}_{(1)} \rightarrow \mathcal{O}_{\operatorname{Gr}(k, \mathscr{A}) \times_{X} \operatorname{Gr}(k, \mathscr{A})} \rightarrow \mathcal{O}_{\Delta(\operatorname{Gr}(k, \mathscr{A}) / X)} \rightarrow 0 .
\end{gathered}
$$

Therefore, as $\Lambda^{m}\left(\mathscr{F}_{(1)} \otimes \mathscr{G}_{(1)}\right)=\bigoplus_{|\alpha|=m} \mathscr{F}_{\alpha} \otimes \mathscr{G}_{\alpha^{*}}$ for $1 \leq m \leq k(n-k)$, the sheaf of ideals of the diagonal embedding $\mathcal{O}_{\Delta(\operatorname{Gr}(k, \mathscr{A}) / X)}$ is in the subcategory

$$
\left\langle\pi_{1}^{*} \mathscr{F}_{\alpha} \otimes \pi_{2}^{*} \mathscr{G}_{\alpha^{*}}|0 \leq| \alpha \mid \leq k(n-k)\right\rangle
$$

of $D(\operatorname{Gr}(k, \mathscr{A}) \times \operatorname{Gr}(k, \mathscr{A}))$. By the projection formula, for any $\mathscr{M} \in D(\operatorname{Gr}(k, \mathscr{A}))$ we have $\mathscr{M}=R\left(\pi_{1}\right)_{*}\left(\pi_{2}^{*} \mathscr{M} \otimes \mathcal{O}_{\Delta(\operatorname{Gr}(k, \mathscr{A}) / X)}\right)$.

To finish the proof, it is enough to verify that

$$
R\left(\pi_{1}\right)_{*}\left(\pi_{2}^{*} \mathscr{M} \otimes\left(\pi_{1}^{*} \mathscr{F}_{\alpha} \otimes \pi_{2}^{*} \mathscr{G}_{\alpha^{*}}\right)\right) \in S(\alpha):
$$

since we have $R\left(\pi_{1}\right)_{*}\left(\pi_{2}^{*} \mathscr{M} \otimes\left(\pi_{1}^{*} \mathscr{F}_{\alpha} \otimes \pi_{2}^{*} \mathscr{G}_{\alpha^{*}}\right)\right)=R\left(\pi_{1}\right)_{*}\left(\pi_{2}^{*}\left(\mathscr{M} \otimes \mathscr{G}_{\alpha^{*}}\right)\right) \otimes \mathscr{F}_{\alpha}$, this is isomorphic to

$$
q^{*}\left(R q_{*}\left(\mathscr{M} \otimes S^{\alpha^{*}} \mathscr{T}\right)\right) \otimes S^{\alpha} \mathscr{R} \text { over } \operatorname{Gr}(k, \mathscr{E}) .
$$

Let $1 \leq k_{1}<\cdots<k_{m}<n$ be a sequence of integers. Given a sheaf of Azumaya algebras $\mathscr{A}$ of rank $n^{2}$ over $X$, we denote by $\mathrm{Fl}\left(k_{1}, \ldots, k_{m}, \mathscr{A}\right)$ the functor defined by $(Y \stackrel{\phi}{\rightarrow} X) \mapsto$ the set of sheaves of left ideals $\mathscr{I}_{1} \subset \cdots \subset \mathscr{I}_{m}$ of $\phi^{*} \mathscr{A}$ such that $\phi^{*} \mathscr{A} / \mathscr{I}_{i}$ is a locally free $\mathscr{O}_{Y}$-module of rank $n\left(n-k_{i}\right)$. 
As in the grassmannian case, we have an étale covering $i: U \rightarrow X$ and a locally free sheaf $\mathscr{E}$ of rank $n$ over $U$ with the following pullback diagram:

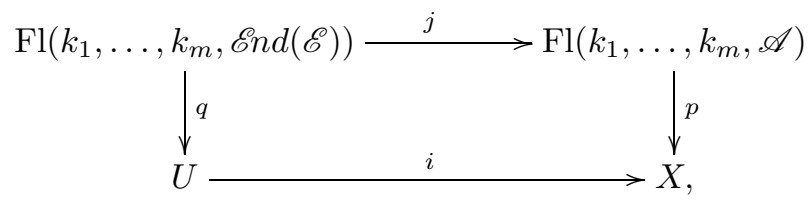

where $\operatorname{Fl}\left(k_{1}, \ldots, k_{m}, \mathscr{E} n d(\mathscr{E})\right)$ is identified with $\mathrm{Fl}\left(k_{1}, \ldots, k_{m}, \mathscr{E}\right)$. We have the tautological flags

$$
\mathscr{R}_{1} \hookrightarrow \cdots \hookrightarrow \mathscr{R}_{m} \hookrightarrow q^{*} \mathscr{E} \rightarrow \mathscr{T}_{1} \rightarrow \cdots \rightarrow \mathscr{T}_{m},
$$

where $\operatorname{rank}\left(\mathscr{R}_{i}\right)=k_{i}$ and $\operatorname{rank}\left(\mathscr{T}_{i}\right)=n-k_{i}$.

Let $\alpha(1), \ldots, \alpha(m-1), \alpha(m)$ be partitions of the forms

$$
\left(\alpha_{1}, \ldots, \alpha_{k_{1}}\right), \ldots,\left(\alpha_{1}, \ldots, \alpha_{k_{m-1}}\right),\left(\alpha_{1}, \ldots, \alpha_{k_{m}}\right)
$$

with $0 \leq \alpha_{i} \leq k_{2}-k_{1}, \ldots, 0 \leq \alpha_{i} \leq k_{m}-k_{m-1}, 0 \leq \alpha_{i} \leq n-k_{m}$, respectively. For $1 \leq i \leq m$, we define $S(\alpha(1), \ldots, \alpha(m))$ to be the full subcategory of $\mathrm{D}\left(\mathrm{Fl}\left(k_{1}, \ldots, k_{m}, \mathscr{A}\right)\right)$ generated by $\mathscr{M}$ in $\mathrm{D}\left(\mathrm{Fl}\left(k_{1}, \ldots, k_{m}, \mathscr{A}\right)\right)$ satisfying

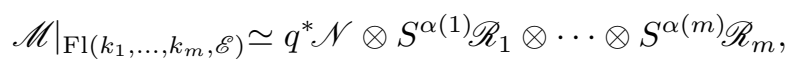

for some $\mathscr{N} \in \mathrm{D}(U)$.

Corollary 4.3. Let $1 \leq k_{1}<\cdots<k_{m}<n$ be a sequence of integers and let $(S(\alpha(1), \ldots, \alpha(m)) \mid$ all partitions of the form $\alpha(i), 1 \leq i \leq m)$ be a sequence of the full subcategories of $\mathrm{D}\left(\mathrm{Fl}\left(k_{1}, \ldots, k_{m}, \mathscr{A}\right)\right)$ in lexicographical order. Then this gives a semiorthogonal decomposition of $\mathrm{D}\left(\mathrm{Fl}\left(k_{1}, \ldots, k_{m}, \mathscr{A}\right)\right)$.

Proof. We shall prove by induction on $m$. The case $m=1$ follows from Theorem 4.2. Assume that the result holds for $m-1$. There are projections

$$
\mathrm{Fl}\left(k_{1}, \ldots, k_{m}, \mathscr{E}\right) \stackrel{q_{m}}{\longrightarrow} \cdots \stackrel{q_{2}}{\longrightarrow} \operatorname{Fl}\left(k_{m}, \mathscr{E}\right) \stackrel{q_{1}}{\longrightarrow} U
$$

and

$$
\mathrm{Fl}\left(k_{1}, \ldots, k_{m}, \mathscr{A}\right) \stackrel{p_{m}}{\longrightarrow} \cdots \stackrel{p_{2}}{\longrightarrow} \mathrm{Fl}\left(k_{m}, \mathscr{A}\right) \stackrel{p_{1}}{\longrightarrow} X .
$$

Let $\mathscr{R}_{2}^{\prime} \subset\left(q_{1} \circ \cdots \circ q_{m-1}\right)^{*} \mathscr{E}$ be the tautological subsheaf over $\operatorname{Fl}\left(k_{2}, \ldots, k_{m}, \mathscr{E}\right)$ and let $\mathscr{A}^{\prime}$ be the sheaf of Azumaya algebra over $\operatorname{Fl}\left(k_{2}, \ldots, k_{m}, \mathscr{A}\right)$ from $\mathscr{E} n d\left(\mathscr{R}_{2}^{\prime}\right)$ by descent. Then, we have $\operatorname{Fl}\left(k_{1}, \ldots, k_{m}, \mathscr{E}\right)=\operatorname{Gr}_{\mathrm{Fl}\left(k_{2}, \ldots, k_{m}, \mathscr{E}\right)}\left(k_{1}, \mathscr{R}_{2}^{\prime}\right)$ and $\operatorname{Fl}\left(k_{1}, \ldots, k_{m}, \mathscr{A}\right)=\operatorname{Gr}_{\mathrm{Fl}\left(k_{2}, \ldots, k_{m}, \mathscr{A}\right)}\left(k_{1}, \mathscr{A}^{\prime}\right)$. Now the result follows from the proofs of Lemma 4.1 and Theorem 4.2

\section{ACKNOWLEDGMENTS}

This work was partially supported by an internal fund from KAIST, TJ Park Junior Faculty Fellowship of POSCO TJ Park Foundation, and National Research Foundation of Korea (NRF) funded by the Minsitry of Science, ICT and Future Planning (2013R1A1A1010171). 


\section{REFERENCES}

[1] A. A. Beullinson, Coherent sheaves on $\mathbf{P}^{n}$ and problems in linear algebra (Russian), Funktsional. Anal. i Prilozhen. 12 (1978), no. 3, 68-69. MR.509388 (80c:14010b)

[2] Marcello Bernardara, A semiorthogonal decomposition for Brauer-Severi schemes, Math. Nachr. 282 (2009), no. 10, 1406-1413, DOI 10.1002/mana.200610826. MR2571702 (2010k:14021)

[3] A. I. Bondal, Representations of associative algebras and coherent sheaves (Russian), Izv. Akad. Nauk SSSR Ser. Mat. 53 (1989), no. 1, 25-44; English transl., Math. USSR-Izv. 34 (1990), no. 1, 23-42. MR992977 (90i:14017)

[4] A. I. Bondal and M. M. Kapranov, Representable functors, Serre functors, and reconstructions (Russian), Izv. Akad. Nauk SSSR Ser. Mat. 53 (1989), no. 6, 1183-1205, 1337; English transl., Math. USSR-Izv. 35 (1990), no. 3, 519-541. MR1039961 (91b:14013)

[5] M. M. Kapranov, On the derived categories of coherent sheaves on some homogeneous spaces, Invent. Math. 92 (1988), no. 3, 479-508, DOI 10.1007/BF01393744. MR939472 (89g:18018)

[6] D. O. Orlov, Projective bundles, monoidal transformations, and derived categories of coherent sheaves (Russian, with Russian summary), Izv. Ross. Akad. Nauk Ser. Mat. 56 (1992), no. 4, 852-862, DOI 10.1070/IM1993v041n01ABEH002182; English transl., Russian Acad. Sci. Izv. Math. 41 (1993), no. 1, 133-141. MR1208153 (94e:14024)

[7] Marc Levine, V. Srinivas, and Jerzy Weyman, K-theory of twisted Grassmannians, K-Theory 3 (1989), no. 2, 99-121, DOI 10.1007/BF00533374. MR1029954 (91b:14007)

[8] I. A. Panin, Algebraic K-theory of Grassmannian manifolds and their twisted forms (Russian), Funktsional. Anal. i Prilozhen. 23 (1989), no. 2, 71-72, DOI 10.1007/BF01078789; English transl., Funct. Anal. Appl. 23 (1989), no. 2, 143-144. MR1011365 (90m:14010)

Department of Mathematical Sciences, KAist, 291 Daehak-ro, Yuseong-gu, Daejeon, 305-701, RePublic OF KoreA

E-mail address: sanghoonbaek@kaist.ac.kr 